\title{
Real-time monitoring of Levy flights in a single quantum system
}

\section{Journal Article}

\section{Author(s):}

Issler, Mena E.; Höller, J.; Imamoglu, Atac

Publication date:

2016-02-15

\section{Permanent link:}

https://doi.org/10.3929/ethz-b-000114196

\section{Rights / license:}

In Copyright - Non-Commercial Use Permitted

\section{Originally published in:}

Physical Review B 93(8), https://doi.org/10.1103/PhysRevB.93.081414

\section{Funding acknowledgement:}

159196 - Quantum interface between solid-state spins, single photon pulses and phonons (SNF) 


\title{
Real-time monitoring of Lévy flights in a single quantum system
}

\author{
M. Issler, J. Höller, and A. Imamoğlu \\ Institute of Quantum Electronics, ETH Zurich, CH-8093 Zurich, Switzerland \\ (Received 6 May 2015; revised manuscript received 10 August 2015; published 29 February 2016)
}

\begin{abstract}
Lévy flights are random walks where the dynamics is dominated by rare events. Even though they have been studied in vastly different physical systems, their observation in a single quantum system has remained elusive. Here we analyze a periodically driven open central spin system and demonstrate theoretically that the dynamics of the spin environment exhibits Lévy flights. For the particular realization in a single-electron charged quantum dot driven by periodic resonant laser pulses, we use Monte Carlo simulations to confirm that the long waiting times between successive nuclear spin-flip events are governed by a power-law distribution; the corresponding exponent $\eta=-3 / 2$ can be directly measured in real time by observing the waiting time distribution of successive photon emission events. Remarkably, the dominant intrinsic limitation of the scheme arising from nuclear quadrupole coupling can be minimized by adjusting the magnetic field or by implementing spin echo.
\end{abstract}

DOI: 10.1103/PhysRevB.93.081414

Lévy flights are widely used to describe anomalous (nonGaussian) diffusion processes. Mathematically, Lévy flights are continuous-time random walks where either the waiting time $\Delta t$ or the step length $\Delta x$ distribution shows an asymptotic power-law dependence $P(\Delta t) \sim \Delta t^{\eta}$ or $P(\Delta x) \sim \Delta x^{\eta}$, with an exponent $-2<\eta<-1$. The fat tail of the distribution leads to diverging average waiting times or average step lengths and the corresponding extreme events dominate the dynamics [1]. Lévy flights have been observed experimentally in various physical systems, such as light scattering in specially engineered materials [2] or in subrecoil laser cooling [3]. In all experiments to date, Lévy dynamics had to be inferred from ensemble properties; tracking Lévy flights of a single quantum system in real time has so far remained elusive.

In this Rapid Communication, we theoretically investigate an open central spin system driven by periodic laser pulses and show that the polarization of the spin environment undergoes anomalous diffusion that can be characterized by Lévy flights. To be specific, we derive the dynamics for a particular realization of the central spin model: an electron confined in a single self-assembled quantum dot (QD) that interacts with the nuclear spin environment. This choice is motivated by striking experimental results on an ensemble of self-assembled QDs [4-8], which we show in this Rapid Communication to be compatible with Lévy flights of the Overhauser field. The random walker in this central spin system corresponds to the nuclear Overhauser field $B_{\mathrm{Oh}}$, the step length to the shift in the Overhauser field $\Delta B_{\mathrm{Oh}}$ induced by a nuclear spin flip and the waiting time is given by the time interval $\Delta t_{\text {nuc }}$ between two successive nuclear spin flips. Even though direct nuclear spin flips in this system are energetically suppressed, spontaneous emission from the laser excited central (electron) spin enables higher order spin-flip processes. We find that long nuclear waiting times are characterized by a corresponding reduction in the photon emission rate. We confirm that the waiting time has a power-law distribution with an exponent $\eta=-3 / 2$ leading to extremely long waiting times. These Lévy flights can be observed by monitoring the waiting time distribution of successive photon detection events. Owing to the favorable ratio of the rates of emitted photons to nuclear spin flips, many photons are scattered between two successive nuclear spin flips which makes it experimentally feasible to probe the nuclear spin dynamics in real time even with a modest photon detection efficiency.

In a previous work, it was proposed that in solidstate emitters under two-color continuous-wave excitation in the coherent population trapping configuration, the hyperfine flip-flop processes give rise to anomalous diffusion in the nuclear spin environment [9]. Despite experimental implementation in nitrogen-vacancy centers [10], the realization of the scheme [9] in self-assembled QDs is hindered by the presence of large anisotropic strain. The latter leads to strong quadrupolar interactions that substantially alter the assumed diffusive model [11-13], masking the underlying nuclear Lévy flights. While the previous work emphasized anomalous diffusion as a tool to achieve cooling of the nuclear spin distribution, the present scheme focuses on monitoring the Lévy flights in the nuclear spin environment. To this end, the directional nuclear spin polarization effects resulting from strain-induced quadrupolar interactions are suppressed by using pulsed laser excitation. This ensures a primarily diffusive model where the nuclear random walk shows Lévy statistics even in the presence of strong quadrupolar interactions.

We consider an electron in a single self-assembled QD coupled to the radiation field reservoir and illuminated by a $\left(\sigma^{+}\right)$circularly polarized laser pulse train propagating along the QD growth direction $x$. The external magnetic field $\vec{B}=B \hat{z}$ is applied transverse to the light propagation direction (Voigt geometry) as shown in Fig. 1(a). The electronic ground states $\left(\left|\uparrow_{x}\right\rangle,\left|\downarrow_{x}\right\rangle\right)$ and the excited, negatively charged trion states $\left(|\uparrow \downarrow \uparrow x\rangle,\left|\uparrow \downarrow \Downarrow_{x}\right\rangle\right)$ realize a four-level system with ground (e) and excited (t) states precessing in the external magnetic field $B$ with frequencies $\omega_{\mathrm{e}}=g_{\mathrm{e}} \mu_{\mathrm{B}} B$ and $\omega_{\mathrm{t}}=$ $g_{\mathrm{h}} \mu_{\mathrm{B}} B$, respectively. The effective $g$ factors of the electron (hole) are given by $g_{\mathrm{e}}<0\left(g_{\mathrm{h}}>0\right)$, and $\mu_{\mathrm{B}}$ is the Bohr magneton. We describe the system in the basis of the light propagation direction and hereafter suppress the subscript $x$ on the basis states, i.e., $|\uparrow x\rangle=|\uparrow\rangle$. The Hamiltonian is then 
(a)

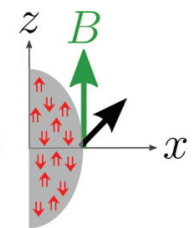

(b)

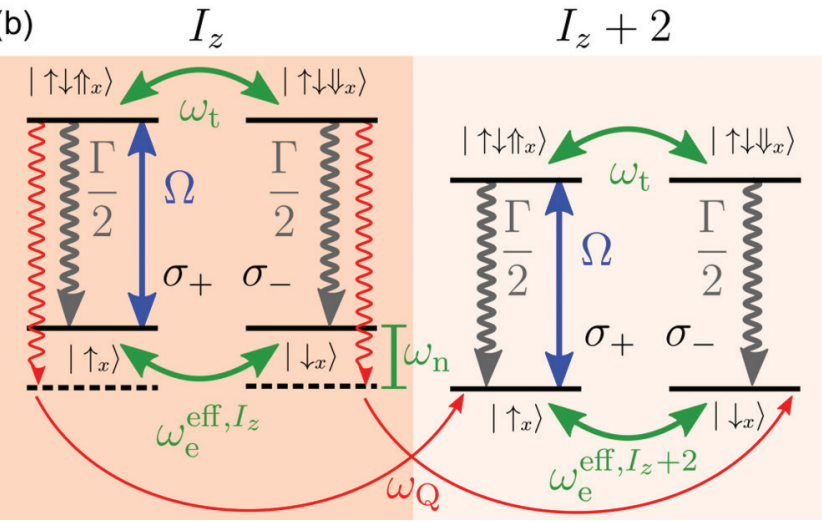

FIG. 1. (a) The magnetic field $B$ is applied in the $z$ direction and the laser pulse train propagates along the $x$ direction, which is the QD growth axis. (b) The electron and the trion precess in the external magnetic field (green) and the $\sigma_{+}$-polarized laser only couples to $\left|\uparrow \uparrow_{x}\right\rangle$ (blue). Spontaneous emission is measured in the circularly polarized basis (gray). On one hand, the coupling to the nuclear spin environment modifies the electron ground state precession frequency, while on the other hand, the slow nuclear spin dynamics is dominated by optically assisted nuclear spin flips (red).

given by $(\hbar=1)$

$$
\begin{aligned}
\hat{H}_{0}= & +\frac{\omega_{\mathrm{e}}}{2}(|\uparrow\rangle\langle\downarrow|+| \downarrow\rangle\langle\uparrow|) \\
& +\frac{\omega_{\mathrm{t}}}{2}(|\uparrow \downarrow \uparrow\rangle\langle\uparrow \downarrow \Downarrow|+| \uparrow \downarrow \Downarrow\rangle\langle\uparrow \downarrow \uparrow|) \\
& +\omega_{\mathrm{te}}(|\uparrow \downarrow \uparrow\rangle\langle\uparrow \downarrow \uparrow|+| \uparrow \downarrow \Downarrow\rangle\langle\uparrow \downarrow \Downarrow|),
\end{aligned}
$$

where $\omega_{\text {te }}$ corresponds to the optical transition energy.

A $\sigma_{+}$-polarized laser pulse couples the states $|\uparrow\rangle$ and $|\uparrow \downarrow \uparrow\rangle$ [12] while leaving $|\downarrow\rangle$ unaffected. Hence, $|\uparrow\rangle$ is called bright and $|\downarrow\rangle$ dark state. The duration of the laser pulse $t_{\mathrm{p}}$ is taken to be short enough so that the coherent evolution due to the four-level Hamiltonian $\hat{H}_{0}$ and spontaneous emission are negligible during the pulse. We justify later that all elements of the electron density matrix $\hat{\rho}$ related to the excited states are zero at the arrival of a pulse. Then the populations before (i) and after (f) a single pulse are given by [14]

$$
\begin{aligned}
\rho_{\uparrow, \uparrow}^{\mathrm{f}} & =\frac{1}{2} \rho_{\uparrow, \uparrow}^{\mathrm{i}}(1+\cos \theta), \\
\rho_{\uparrow \downarrow \uparrow, \uparrow \downarrow \uparrow}^{\mathrm{f}} & =\frac{1}{2} \rho_{\uparrow, \uparrow}^{\mathrm{i}}(1-\cos \theta),
\end{aligned}
$$

where the pulse area $\theta=2 \int \Omega(t) d t$ determines the excited electron population and $\Omega$ is the laser Rabi frequency.

Next we study the dynamics when the $\pi$ pulses arrive periodically with a repetition period $\tau$ in the presence of spontaneous emission at a rate $\Gamma$. If the pulse separation $\tau$ satisfies $\tau \gg \Gamma^{-1}$ the trion population decays completely within the pulse separation $\tau$, which justifies (1). The combined coupling of the four-level system to a periodic laser pulse train and the radiation field reservoir asymptotically forces the electron into a periodic quasisteady state. This occurs by sequential excitation of the bright state by a laser pulse, followed by spontaneous emission of a photon at a rate $\Gamma$ and the accompanying decay into one of the two ground states. The asymptotic bright state population $\rho_{\uparrow, \uparrow}^{\mathrm{i}, *}$ before the pulse is then given by [14]

$$
\rho_{\uparrow, \uparrow}^{\mathrm{i}, *}=\frac{\sin ^{2}\left(\omega_{\mathrm{e}} \tau / 2\right)}{\frac{1}{2}+\sin ^{2}\left(\omega_{\mathrm{e}} \tau / 2\right)}
$$

provided $\theta=\pi$ and $\omega_{\mathrm{e}} \gg \Gamma$. Similarly, the coarse-grained spontaneous emission rate is given by

$$
\begin{aligned}
\Gamma_{\text {spon }} & =\frac{1}{\tau} \int_{0}^{\tau} \frac{\Gamma}{2}\left[\rho_{\uparrow \downarrow \uparrow, \uparrow \downarrow \uparrow}(t)+\rho_{\uparrow \downarrow \Downarrow, \uparrow \downarrow \Downarrow \downarrow}(t)\right] d t \\
& =\frac{1}{\tau} \rho_{\uparrow, \uparrow}^{\mathrm{i}, *}=\frac{1}{\tau} \frac{\sin ^{2}\left(\omega_{\mathrm{e}} \tau / 2\right)}{\frac{1}{2}+\sin ^{2}\left(\omega_{\mathrm{e}} \tau / 2\right)} .
\end{aligned}
$$

If the bright state population at the arrival of the pulse vanishes, so does the optical absorption and hence the coarsegrained spontaneous emission rate $\Gamma_{\text {spon }}$. This occurs exactly if the electron precession frequency satisfies the synchronization condition

$$
\omega_{\mathrm{e}} \in \frac{2 \pi}{\tau} \mathbb{Z} .
$$

The expansion of $\Gamma_{\text {spon }}$ close to a synchronization condition (2) with $m \in \mathbb{Z}$ gives

$$
\Gamma_{\text {spon }}=\frac{\tau}{2}\left(\omega_{\mathrm{e}}-\frac{2 \pi}{\tau} m\right)^{2}+O\left[\left(\omega_{\mathrm{e}}-\frac{2 \pi}{\tau} m\right)^{4}\right] .
$$

The fact that $\Gamma_{\text {spon }}$ depends quadratically on $\omega_{\mathrm{e}}$ is essential for the emergence of Lévy flights.

To derive the dynamics of the spin environment, the relevant interactions of the central spin with the spin environment need to be identified, which depend on the realization of the central spin model. While the following discussion focuses on the dominant interactions in self-assembled QDs, i.e., the Fermi-contact hyperfine interaction and strain-induced quadrupolar couplings [12], our scheme is not limited to these specific interactions. The Fermi-contact hyperfine interaction between the QD electron and the nuclear spin environment constitutes the coupling of the central spin to the nuclear spin environment and is given by $[12,15,16]$ $\hat{H}_{\mathrm{hf}}=\sum_{j=1}^{N} A_{j} \hat{S}_{z} \hat{I}_{z}^{j}+\sum_{j=1}^{N} \frac{A_{j}}{2}\left(\hat{S}_{-} \hat{I}_{+}^{j}+\hat{S}_{+} \hat{I}_{-}^{j}\right)$. The number of nuclear spins is $N \approx 10^{4}-10^{6}$ and $\hat{I}_{z}^{j}$ is the spin projection operator to the $z$ axis of the $j$ th nucleus for $1 \leqslant j \leqslant N$. The hyperfine coupling constant $A_{j}$ depends on the overlap of the electron wave function with the $j$ th nucleus. Equal coupling of all nuclei to the electron leads to the homogeneous hyperfine coupling constant $A=\frac{A_{\mathrm{H}}}{N}=\frac{1}{N} \sum_{j=1}^{N} A_{j}$, where $A_{\mathrm{H}}$ is the hyperfine coupling constant of the material, assuming a single nuclear species. The first term of $\hat{H}_{\mathrm{hf}}$, which we denote by $\hat{H}_{\mathrm{Oh}}$, describes the Overhauser shift [12]; the electron precesses around the vector sum of the external field and the effective magnetic Overhauser field $B_{\mathrm{Oh}}=\sum_{j=1}^{N} A_{j}\left\langle\hat{I}_{z}^{j}\right\rangle /\left(g_{\mathrm{e}} \mu_{\mathrm{B}}\right)$, created by the nuclear spins. The associated Overhauser precession frequency is $\omega_{\mathrm{Oh}}=\sum_{j=1}^{N} A_{j}\left\langle\hat{I}_{z}^{j}\right\rangle$. The second term of $\hat{H}_{\mathrm{hf}}$ describes nuclear spin flip-flop processes that are suppressed at high magnetic fields due to the energy mismatch 
between the electron Zeeman energy $\left(\omega_{\mathrm{e}}\right)$ and the average nuclear Zeeman energy $\left(\omega_{\mathrm{n}}\right)$, i.e., $\omega_{\mathrm{e}} \gg \omega_{\mathrm{n}}[12]$.

In self-assembled QDs, the quadrupolar interaction in conjunction with the Overhauser field term constitutes the dominant mechanism for diffusive spontaneous-emission assisted nuclear spin flips; we therefore neglect the flip-flop process from here on. We emphasize, however, that the scheme is also applicable when the dominant nuclear interaction is not given by the quadrupolar interaction but instead by the flip-flop processes in $\hat{H}_{\mathrm{hf}}$, an effective noncollinear interaction of the form $\hat{H}_{\mathrm{nc}}=\sum_{j} A_{\mathrm{nc}} \hat{S}_{z} \hat{I}_{x}^{j}$ [11-13] or a hole-induced noncollinear process [17]. Given that the dominant quadrupolar interactions in self-assembled QDs stem from electric field gradients that primarily point along the $x$ axis, the dominant nuclear spin dynamics in self-assembled QDs is described by the Hamiltonians

$$
\begin{aligned}
\hat{H}_{\mathrm{n}} & =-\sum_{j=1}^{N} \omega_{\mathrm{n}}^{j} \hat{I}_{z}^{j}, \\
\hat{H}_{\mathrm{Oh}} & =\sum_{j=1}^{N} A_{j} \hat{S}_{z} \hat{I}_{z}^{j}, \\
\hat{H}_{\mathrm{Q}} & =\sum_{j=1}^{N} \frac{\omega_{\mathrm{Q}}^{j}}{2}\left[\left(\hat{I}_{x}^{j}\right)^{2}-\frac{I^{j}\left(I^{j}+1\right)}{3} \hat{\mathbb{1}}^{j}\right],
\end{aligned}
$$

where $\omega_{\mathrm{n}}^{j}$ is the Zeeman splitting of the $j$ th nucleus, and $\omega_{\mathrm{Q}}^{j}$ is the quadrupolar energy for $1 \leqslant j \leqslant N$.

The effect of the electron-nuclei interaction on the coupled system is twofold. On the one hand, the electron dynamics is modified by the Overhauser field which changes $\omega_{\mathrm{e}}$ to the effective electron precession frequency $\omega_{\mathrm{e}}^{\text {eff }}=\omega_{\mathrm{e}}+\omega_{\mathrm{Oh}}$. The dark state is then only reached at the arrival of a pulse if synchronization (2) is fulfilled for the effective precession frequency

$$
\omega_{\mathrm{e}}^{\mathrm{eff}} \in \frac{2 \pi}{\tau} \mathbb{Z}
$$

The modified electron dynamics results in a coarse-grained spontaneous emission rate $\Gamma_{\text {spon }}$ that depends on $\omega_{\mathrm{e}}^{\mathrm{eff}}$ and thus on the nuclear Overhauser field $B_{\mathrm{Oh}}$, and vanishes if the synchronization condition is satisfied.

On the other hand, the quadrupolar interaction couples states of different Overhauser field. However, in a strong magnetic field the energy mismatch between such states suppresses nuclear spin flips. In the presence of optical excitation, a spontaneously emitted photon can supply the additional energy which gives rise to a slow time evolution of the Overhauser field through these spontaneous-emission assisted nuclear spin flips. These processes can be derived perturbatively by applying a Schrieffer-Wolff transformation $[9,14,18]$ and are of order $O\left(\epsilon^{2}\right)$ in the small perturbation parameter $\epsilon=\sqrt{N} \frac{A \omega_{\mathrm{Q}}}{32 \omega_{\mathrm{n}}^{2}}$. The spontaneous-emission assisted nuclear spin flips can be visualized as a spontaneous emission of a photon to a virtual state, allowing for a concomitant energy-conserving nuclear spin flip [Fig. 1(b)] and occurring
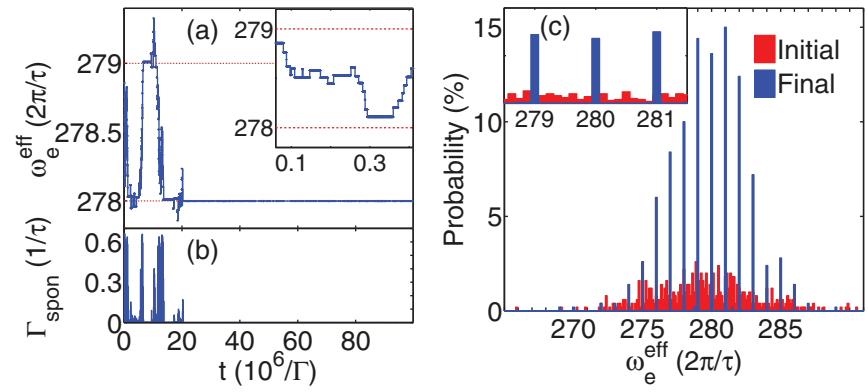

FIG. 2. (a) The random walk of the effective electron precession frequency (blue) spends most of the time close to traps (red), indicating a Lévy distribution for long waiting times. The inset resolves the random walk between the traps. (b) $\Gamma_{\text {spon }}$ gives the spontaneous emission rate of the corresponding random walk in (a). (c) Exposing an ensemble of 500 QDs with normally distributed initial nuclear polarization $\left(\left\langle I_{z}\right\rangle=5, \sigma=\sqrt{N}\right)$ (red) to the pulsed laser train for $10^{8} \tau=10^{9} / \Gamma$ leads to a final (blue) distribution of the effective electron precession frequency that is heavily peaked at the different traps (inset). For all simulations we used the parameters $\tau=10 / \Gamma, \omega_{\mathrm{e}}=280 \frac{2 \pi}{\tau}, \omega_{\mathrm{n}}=0.18 \Gamma, N=$ $2000, \omega_{\mathrm{Q}}=0.013 \Gamma, A_{\mathrm{H}}=100 \Gamma$ such that $\epsilon \approx 3 \times 10^{-2}$ and we assumed inhomogeneous hyperfine coupling constants.

with a rate [14]

$$
R_{\mathrm{nuc}}=\epsilon^{2} \Gamma_{\text {spon }} \approx \epsilon^{2} \frac{\tau}{2}\left(\omega_{\mathrm{e}}^{\mathrm{eff}}-\frac{2 \pi}{\tau} m\right)^{2}
$$

under the assumptions of homogeneous hyperfine and quadrupolar coupling, $\omega_{\mathrm{n}} \gg A$ and $I_{z}^{j}= \pm \frac{1}{2}$ for simplicity [19]. The rate of optically assisted nuclear spin flips $R_{\text {nuc }}$ is drastically reduced in the vicinity of a synchronization condition (3). The signatures of this reduction can be observed in a single realization of a random walk of $\omega_{\mathrm{e}}^{\text {eff }}\left(I_{z}\right)$ : the random walk freezes near a synchronization condition which therefore constitutes a trap [red lines in Fig. 2(a)]. An unsynchronized electron is optically excited and induces an unbiased random walk of the nuclear polarization $I_{z}=\sum_{j=1}^{N}\left\langle I_{z}^{j}\right\rangle$ until $\omega_{\mathrm{e}}^{\text {eff }}$ is synchronized. In the absence of other interactions, the time spent near such a trap can be on the order of the measurement time. We also simulated the time evolution of the distribution of $\omega_{\mathrm{e}}^{\mathrm{eff}}$ starting from a Gaussian distribution of $\left\{I_{z}^{j}\right\}_{j=1}^{N}$ [red histogram in Fig. 2(c)]. We find that the application of a periodic laser pulse train drastically cools the nuclear spins; the probability of finding $\omega_{\mathrm{e}}^{\mathrm{eff}}$ within a single nuclear spin flip from a trap (3) exceeds $99 \%$. These simulations are in excellent qualitative agreement with experiments carried out on an ensemble of single negatively charged self-assembled QDs [5]. Since we show below that the dynamics leading to the narrowed nuclear spin distribution obeys Lévy statistics, the experimental results $[4,5]$ are likely to be compatible with nuclear Lévy flights.

We analyze the asymptotic nuclear waiting time distribution which allows us to predict Lévy flights in the nuclear spin environment. To this end, we quantify the freezing of the random walk of $\omega_{\mathrm{e}}^{\text {eff }}$ in the vicinity of a trap and introduce the probability distribution of the waiting time between two successive nuclear spin-flip events $P_{\text {nuc }}\left(\Delta t_{\text {nuc }}\right)$ [3]. Clearly, long waiting times occur for $\omega_{\mathrm{e}}^{\text {eff }}$ close to a trap since $\Delta t_{\text {nuc }} \sim$ $R_{\text {nuc }}^{-1} \propto\left(\omega_{\mathrm{e}}^{\text {eff }}-\frac{2 \pi}{\tau} m\right)^{-2}$. This relationship of $\Delta t_{\text {nuc }}$ to $\omega_{\mathrm{e}}^{\text {eff }}$ 


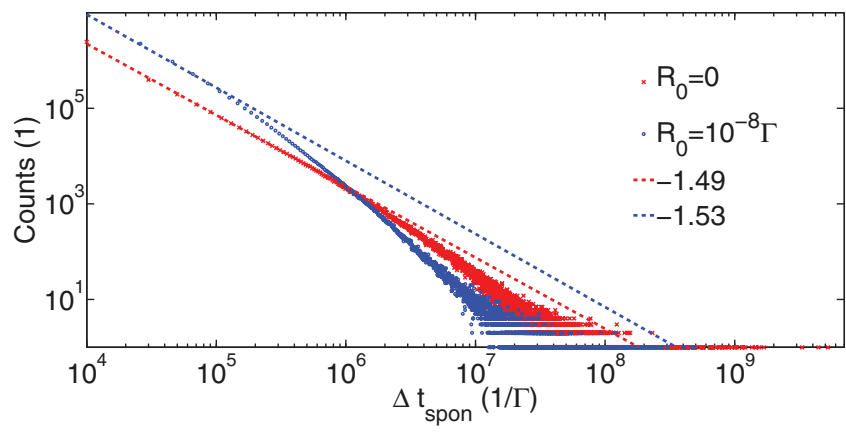

FIG. 3. The optical waiting time distribution for $R_{0}=0$ (red) is extracted from 100 time traces. The corresponding power-law exponent in this simulation is -1.49 , consistent with the theoretical prediction of $-3 / 2$. For $R_{0}^{-1}=10^{8} / \Gamma$ (blue) we find a regime where a power law with exponent -1.53 is observed, followed by an exponential decay. All other parameters are the same as in Fig. 2.

allows us to write $P_{\text {nuc }}\left(\Delta t_{\text {nuc }}\right) d \Delta t_{\text {nuc }}=\Pi_{\text {nuc }}\left(\omega_{\mathrm{e}}^{\text {eff }}\right) d \omega_{\mathrm{e}}^{\text {eff }}$, where the probability to reach an effective precession frequency $\omega_{\mathrm{e}}^{\text {eff }}$ in the trap $\Pi_{\text {nuc }}\left(\omega_{\mathrm{e}}^{\text {eff }}\right)$ is assumed to be uniform. Estimating the change in $\Delta t_{\text {nuc }}$ induced by an infinitesimal change in $\omega_{\mathrm{e}}^{\mathrm{eff}}$ via $\frac{d \Delta t_{\text {nuc }}}{d \omega_{\mathrm{e}}^{\text {eff }}} \propto\left(\omega_{\mathrm{e}}^{\text {eff }}-\frac{2 \pi}{\tau} m\right)^{-3} \propto \Delta t_{\text {nuc }}^{3 / 2}$, results in the asymptotic power-law distribution

$$
P_{\text {nuc }}\left(\Delta t_{\text {nuc }}\right) \propto \Delta t_{\text {nuc }}^{-3 / 2} .
$$

This expression is confirmed by a more rigorous derivation [20] or [14] and predicts that the evolution of the nuclear spin environment is governed by Lévy flights. Furthermore, it is valid for $\Delta t_{\text {nuc }} \gg\left(\left.R_{\text {nuc }}\right|_{\omega_{\mathrm{e}}^{\text {eff }}=(2 \pi / \tau) m \pm A}\right)^{-1}[14,21]$ and entails the divergence of the expectation value of this distribution. The Lévy behavior of the nuclear spin-flip waiting time is thus based on the quadratic dependence of $\Gamma_{\text {spon }}$ and $R_{\text {nuc }}$ on $\omega_{\mathrm{e}}^{\text {eff }}$.

The physical observable in an experiment is the waiting time $\Delta t_{\text {spon }}$ between two successive photon emission events, irrespective of whether the photon emission is accompanied by a nuclear spin flip or not. The rate of photon emission events is given by $\Gamma_{\text {spon }}$ and an analogous derivation yields the waiting time distribution of successive photon emission events [14]

$$
P_{\text {spon }}\left(\Delta t_{\text {spon }}\right) \propto \Delta t_{\text {spon }}^{-3 / 2},
$$

for $\Delta t_{\text {spon }} \gg \epsilon^{2}\left(\left.R_{\text {nuc }}\right|_{\omega_{\mathrm{e}}^{\text {eff }}=(2 \pi / \tau) m \pm A}\right)^{-1}$. As a consequence of the proportionality of the rate of nuclear spin-flip events $R_{\text {nuc }}$ to spontaneous emission events $\Gamma_{\text {spon }}$, the power-law exponents of the two waiting time distributions $P_{\text {nuc }}$ and $P_{\text {spon }}$ are identical. Since the rate of photon detection events is enhanced by a factor $\epsilon^{-2}$ compared to the nuclear spin-flip rate, it is possible to monitor the Lévy flight behavior of nuclear trapping times in real time even with modest photon detection efficiencies.

To numerically verify the power-law behavior of the waiting time distribution, we averaged over 100 Monte Carlo simulations of the type shown in Fig. 2(a) and extracted a power-law exponent $\eta=-1.49$ (red dotted line in Fig. 3) which is in excellent agreement with the predicted distribution of optical waiting times (5).

Experimentally observable waiting times are limited due to additional interactions that have been neglected so far. To investigate their impact on the probability of nuclear trapping times, we assume that they can be modeled as an additional constant nuclear spin-flip rate $R_{0}$ such that the overall nuclear spin-flip rate becomes

$$
\tilde{R}_{\text {nuc }}=R_{\text {nuc }}+R_{0} .
$$

The constant rate $R_{0}$ prevents diverging average trapping times since it effectively limits them to $R_{0}^{-1}$. This scenario corresponds to a truncated Lévy flight [22]. We derived the modified distribution of nuclear trapping times [14]

$$
\tilde{P}_{\text {nuc }}\left(\Delta t_{\text {nuc }}\right) \propto \Delta t_{\text {nuc }}^{-3 / 2}\left(1+2 R_{0} \Delta t_{\text {nuc }}\right) e^{-R_{0} \Delta t_{\text {nuc }}} .
$$

If $R_{0}=0$, then expression (4) is recovered, but if $\Delta t_{\text {nuc }} \gg R_{0}^{-1}$ the exponential term dominates and leads to an exponential decay. However, in an intermediate regime, where the background rate $R_{0}$ is much smaller than $R_{\text {nuc }}$ at a single spin flip away from synchronization, i.e., $\left.R_{0} \ll R_{\text {nuc }}\right|_{\omega_{\mathrm{e}}^{\text {eff }}=m(2 \pi / \tau)+A}$, a power-law regime is observed before the exponential cutoff. The optical waiting time distribution $\tilde{P}_{\text {spon }}$ with constant offset $R_{0}$ is again closely related to the nuclear trapping time distribution $\tilde{P}_{\text {nuc }}$. A model simulation including a constant offset $R_{0} \neq 0$ shows a power-law distribution with coefficient -1.53 (blue dotted line in Fig. 3) for intermediate waiting times and an exponential decay for large waiting times. The physical origin of $R_{0}$ can be classified as system specific or intrinsic to the scheme. Whereas the former can be suppressed by proper engineering, the intrinsic limitation stems from the coherent rotation between different $I_{z}^{j}$ eigenstates in the dark period due to the quadrupolar interaction itself. Even though the energy mismatch between these states prevents actual nuclear spin flips, the laser pulse terminating the period of coherent evolution under $H_{\mathrm{Q}}$ can effect a measurement interaction and could lead to a nuclear spin flip. This physical mechanism and one possibility to minimize the corresponding contribution to $R_{0}$ is discussed in [14].

In summary, we have demonstrated that the polarization of the spin environment of a central spin system that is driven by a resonant periodic pulse train exhibits Lévy flights. Thanks to the decoupling of the central spin and the environment and the proportionality of the environmental spin flip and spontaneous emission rates, our calculations indicate that the signatures of Lévy flights can be observed by measuring the waiting time distribution of the emitted photons. While we illustrated the scheme with self-assembled quantum dots, we expect our findings to be relevant for a broad class of solid-state emitters including nitrogen-vacancy centers. More generally, our results show that the previously established connection between Lévy flights and dark states [3,9] can be extended to systems with explicit time dependence.

This work is funded by the Swiss National Science Foundation (SNSF) under Grant No. 200020-159196. 
[1] M. F. Shlesinger, G. M. Zaslavsky, and J. Klafter, Nature (London) 363, 31 (1993).

[2] P. Barthelemy, J. Bertolotti, and D. S. Wiersma, Nature (London) 453, 495 (2008).

[3] F. Bardou, J.-P. Bouchaud, O. Emile, A. Aspect, and C. CohenTannoudji, Phys. Rev. Lett. 72, 203 (1994).

[4] A. Greilich, R. Oulton, E. A. Zhukov, I. A. Yugova, D. R. Yakovlev, M. Bayer, A. Shabaev, A. L. Efros, I. A. Merkulov, V. Stavarache, D. Reuter, and A. Wieck, Phys. Rev. Lett. 96, 227401 (2006).

[5] A. Greilich, A. Shabaev, D. R. Yakovlev, A. L. Efros, I. A. Yugova, D. Reuter, A. D. Wieck, and M. Bayer, Science (New York, N.Y.) 317, 1896 (2007).

[6] E. Barnes and S. E. Economou, Phys. Rev. Lett. 107, 047601 (2011).

[7] S. E. Economou and E. Barnes, Phys. Rev. B 89, 165301 (2014).

[8] I. A. Yugova, M. M. Glazov, E. L. Ivchenko, and A. L. Efros, Phys. Rev. B 80, 104436 (2009).

[9] M. Issler, E. M. Kessler, G. Giedke, S. Yelin, I. Cirac, M. D. Lukin, and A. Imamoglu, Phys. Rev. Lett. 105, 267202 (2010).

[10] E. Togan, Y. Chu, A. Imamoglu, and M. D. Lukin, Nature (London) 478, 497 (2011).

[11] A. Högele, M. Kroner, C. Latta, M. Claassen, I. Carusotto, C. Bulutay, and A. Imamoglu, Phys. Rev. Lett. 108, 197403 (2012).

[12] B. Urbaszek, X. Marie, T. Amand, O. Krebs, P. Voisin, P. Maletinsky, A. Högele, and A. Imamoglu, Rev. Mod. Phys. 85, 79 (2013).
[13] C. Bulutay, Phys. Rev. B 85, 115313 (2012).

[14] See Supplemental Material at http://link.aps.org/supplemental/ 10.1103/PhysRevB.93.081414 for details on the dynamics of the coupled open system that we consider and a more detailed derivation of the trapping time distribution using Levy flights. Additional simulations presented there validate our approach when including various limitations.

[15] I. T. Vink, K. C. Nowack, F. H. L. Koppens, J. Danon, Y. V. Nazarov, and L. M. K. Vandersypen, Nat. Phys. 5, 764 (2009).

[16] C. Latta, A. Högele, Y. Zhao, A. N. Vamivakas, P. Maletinsky, M. Kroner, J. Dreiser, I. Carusotto, A. Badolato, D. Schuh, W. Wegscheider, M. Atature, and A. Imamoglu, Nat. Phys. 5, 758 (2009).

[17] W. Yang and L. J. Sham, Phys. Rev. B 85, 235319 (2012).

[18] I. Shavitt and L. T. Redmon, J. Chem. Phys. 73, 5711 (1980).

[19] $R_{\text {nuc }}$ is derived for spin- $\frac{1}{2}$ particles by replacing $\left(I_{ \pm}^{j}\right)^{2} \rightarrow I_{ \pm}^{j}$; this captures the relevant nuclear dynamics.

[20] F. Bardou, J.-P. Bouchaud, A. Aspect, and C. Cohen-Tannoudji, Lévy Statistics and Laser Cooling (Cambridge University Press, Cambridge, 2003).

[21] For $\Pi_{\text {nuc }}\left(\omega_{\mathrm{e}}^{\text {eff }}\right)$ to be uniform, the distance to the synchronization condition should not exceed a single nuclear spin flip $A$, where the waiting time is given by $\tau_{1}$. Therefore, (4) only describes the tail of the distribution, i.e., $\Delta t_{\text {nuc }} \gg \tau_{1}$.

[22] R. N. Mantegna and H. E. Stanley, Phys. Rev. Lett. 73, 2946 (1994). 